\title{
A New Technique for RF Distribution
}

\section{Robyn Madrak $^{a *}$ and David Wildman ${ }^{a}$}

${ }^{a}$ Fermi National Accelerator Lab,

P. O. Box 500, Batavia, IL, U.S.A.

E-mail: madrakefnal.gov

\begin{abstract}
For independent phase and amplitude control, RF cavities are often driven by one power source per cavity. In many cases it would be advantageous in terms of cost to instead use one higher power source for many cavities. Vector modulators have been developed, which, when used with a single source provide for the independent phase and amplitude control which would have been otherwise lost. The key components of these vector modulators are a novel type of phase shifter - adjustable fast phase shifters with perpendicularly biased garnets. The vector modulators have been constructed and used with a single klystron in a $3.4 \mathrm{MeV}$ test linac to successfully accelerate proton beam.
\end{abstract}

KEYWORDS: Accelerator Subsystems and Technologies, Acceleration cavities.

${ }^{*}$ Corresponding author. 


\section{Contents}

1. Introduction 囵

2. Vector Modulators 囵

3. Phase Shifters 3

3.1 Low Power Measurements

3.1.1 Phase Shift and Losses

3.1.2 Effective Permeability 5

3.1.3 Response Time 11

4. Vector Modulator High Power Operation 12

4.1 Preparation 12

4.2 Standalone Operation 13

4.3 Vector Modulators with Beam in The Six Cavity Test

5. Summary

\section{Introduction}

A new type of $325 \mathrm{MHz}$ RF vector modulator has been developed. This allows independent phase and amplitude control of RF cavities being driven by a single high power source. Two types of vector modulators (rated at $\approx 500 \mathrm{~kW}$, and one lower power, rated at $\approx 75 \mathrm{~kW}$ ) were initially developed for the High Intensity Neutrino Source (HINS) [1] R\&D test linac at Fermilab. HINS was at first intended to be a $60 \mathrm{MeV}$ pulsed linac with a radiofrequency quadrupole (RFQ) and both superconducting and normal conducting cavities, all being driven by a single $2.5 \mathrm{MW}, 325 \mathrm{MHz}$ klystron. Its purpose was to demonstrate the feasibility of a pulsed $8 \mathrm{GeV}$ linac [2], with $25 \mathrm{~mA}$ of beam current in either $1 \mathrm{~ms}$ pulses at a $10 \mathrm{~Hz}$ rate or $3 \mathrm{~ms}$ pulses at a $2.5 \mathrm{~Hz}$ rate. In this design, one vector modulator is placed between the klystron and each cavity (or RFQ) to independently control the phase and amplitude of the RF drive signal to the cavity's coupling loop.

Before the $60 \mathrm{MeV}$ demonstration linac was completed, the plans for it were scaled back drastically, to a test linac with one radiofrequency quadrupole, two "pillbox" style buncher cavities, and four room temperature crossbar H (RTCH) type [B] cavities. Nevertheless, this 'Six Cavity Test' [ 四, as it was named, provided an opportunity to demonstrate the acceleration of beam with the vector modulators and a single klystron. Though the vector modulators were developed specifically for HINS, the application is broad and they could be useful in other instances.

The key components in these vector modulators are adjustable, high power phase shifters. The first high power microwave phase shifters using ferrite-loaded coaxial structures were first 
described more than forty-five years ago. [5] [6] Recently, there has been renewed interest in the field with advances in technology and materials. The possibility of powering multiple RF cavities from a single RF source has become a viable alternative to the traditional single RF source per cavity [प] [[]].

\section{Vector Modulators}

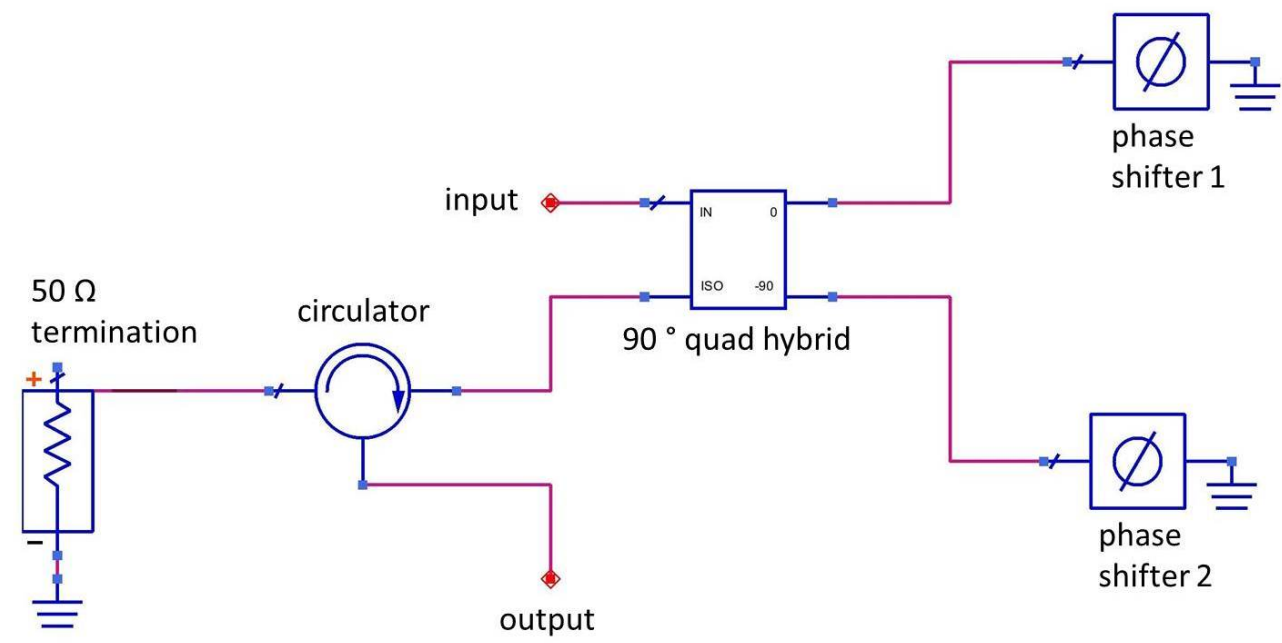

Figure 1. Vector modulator schematic. The input to the hybrid coupler is split between two adjustable phase shifters which are shorted at one end. The output of the coupler combines phase shifted signals. The fact that each of the phase shifters is independently adjustable gives independent phase and amplitude control of the combined signal at the hybrid output.

A schematic of a vector modulator is shown in Fig. 1. A picture of one complete $75 \mathrm{~kW}$ vector modulator is shown in Fig. 2 . The components are a 90 degree hybrid coupler, two independently controlled adjustable phase shifters, a circulator, and $50 \Omega$ load.

The klystron output power is distributed by a WR2300 waveguide. A portion of the RF output is tapped off of the waveguide to port 1 of a $3 \mathrm{~dB}$ quad hybrid. The RF is is equally split with a $90^{\circ}$ phase difference between ports 2 and 3. Two shorted, coaxial, garnet loaded phase shifters, described below, are attached to ports 2 and 3 and provide full reflections with phase shifts $\Delta \phi_{2}$ and $\Delta \phi_{3}$ respectively. The desired phase shift is produced by a variable solenoidal magnetic field along the axis of the coaxial line which is used to adjust the permeability, $\mu$, of the ferrite. These reflected signals are recombined at the output (port 4) of the quad hybrid. The resulting output phase and power are given by

$$
\begin{gathered}
P_{\text {out }}=P_{\max } \cos ^{2}\left(\left(\Delta \phi_{2}-\Delta \phi_{3}\right) / 2\right) \\
\Phi_{\text {out }}=\phi_{0}+\left(\Delta \phi_{2}+\Delta \phi_{3}\right) / 2
\end{gathered}
$$

where $\phi_{0}$ is a constant phase offset. Once the range of possible phase shifts (the range of $\Delta \phi_{2}$ and $\left.\Delta \phi_{3}\right)$ is determined, the range of output power $\left(P_{\text {out }}\right)$ and phase $\left(\Phi_{\text {out }}\right)$ are determined. The output 


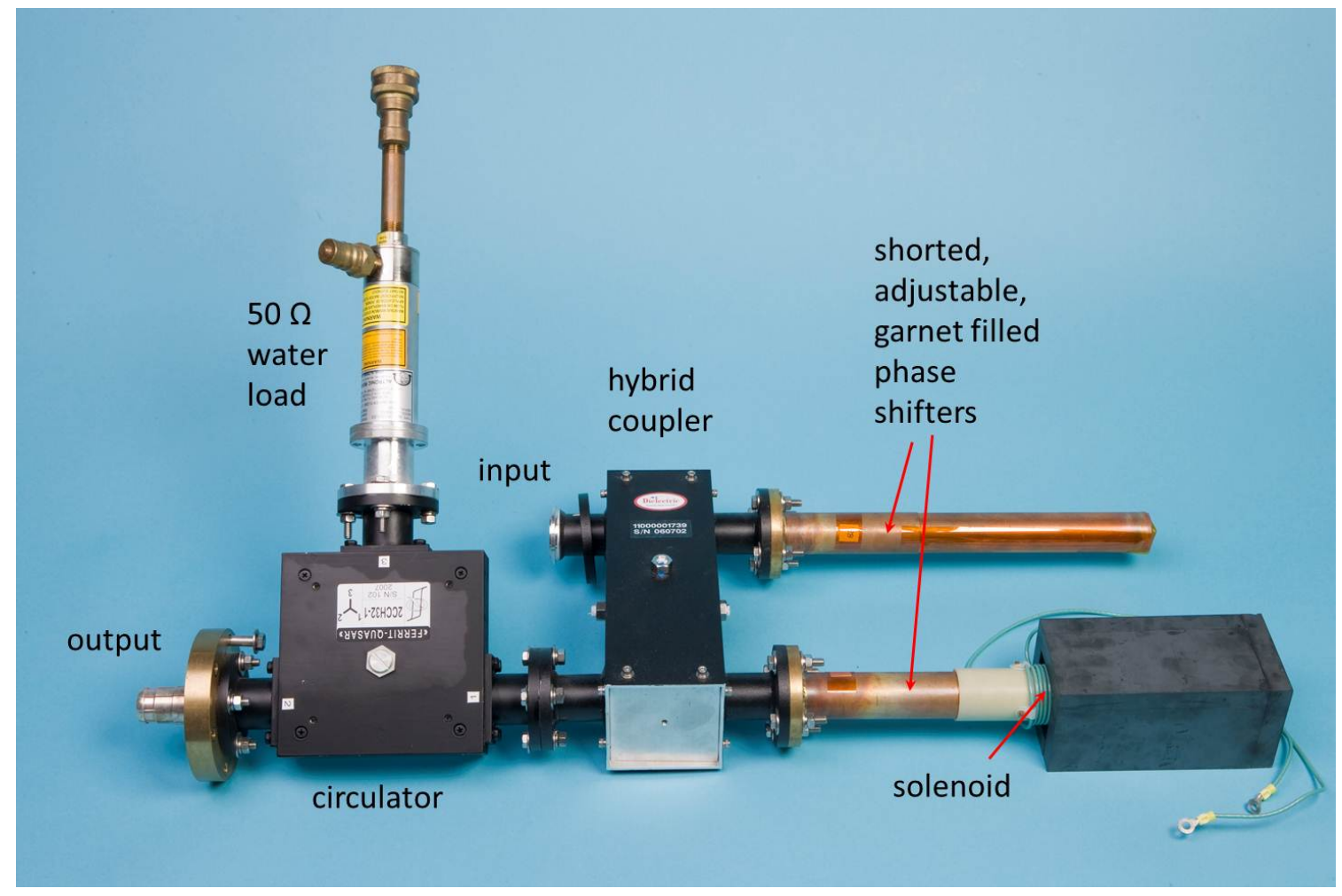

Figure 2. A picture of one complete $75 \mathrm{~kW}$ vector modulator. A solenoid, which is needed for the adjustability, and flux return for it, surround both phase shifters. They have been removed from the top phase shifter in this picture.

range can be shifted by adding a constant phase offset to port 2 or 3 of the hybrid, though this was not necessary here.

For the smaller vector modulator, a three port $75 \mathrm{~kW}$ circulator with a $5 \mathrm{~kW}$ water cooled RF load is connected between the quad hybrid output and the RF cavity to isolate the phase shifters from any power being reflected from the cavity during the cavity filling time or under cavity detuning conditions.

The higher power vector modulator designed to be used with the RFQ is similar to the one described above except that the components are physically larger. The $3 \mathrm{~dB}$ quad hybrid has a coaxial design with 6" EIA flanges and is filled with $\mathrm{SF}_{6}$ to prevent sparking. The two phase shifters are 3.125" OD, garnet loaded, shorted coaxial lines and are also filled with $\mathrm{SF}_{6}$. A high power three port coaxial circulator, rated at $650 \mathrm{~kW}$ for a $4 \mathrm{~ms}$ pulse, is used to prevent the reflection of power back to the phase shifters.

\section{Phase Shifters}

The phase shifters are OFHC copper shorted coaxial transmission lines filled with a $5^{\prime \prime}$ long section of aluminum doped yttrium-iron garnet (TCI Ceramics type AL-400) which has a saturation magnetization $\left(4 \pi M_{s}\right)$ of 400 gauss. Drawings of the two types of phase shifters are shown in Fig. B. 
- For Cavities ( 75 kW):

1.5 "OD X 0.65 "ID X 5"long garnet

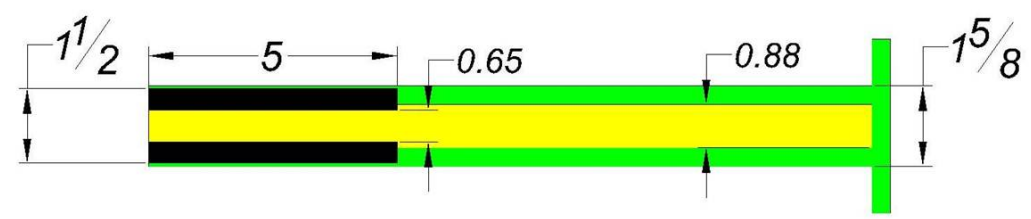

- $\quad$ For RFQ ( 500 kW):

3 "OD X 0.65 "ID X 5"long garnet

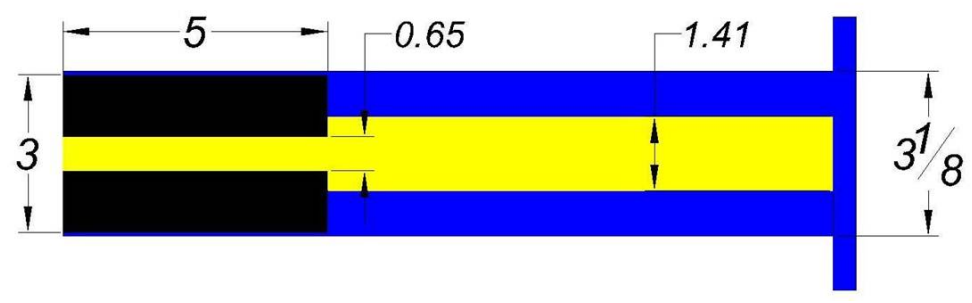

Figure 3. Drawings and dimensions of the two types of phase shifters.

The $75 \mathrm{~kW}$ version uses a $1.5^{\prime \prime}$ OD x $0.65^{\prime \prime}$ ID x $5^{\prime \prime}$ long garnet cylinder at the shorted end over a $0.65^{\prime \prime}$ diameter center conductor. The center conductor diameter is then increased to $0.880^{\prime \prime}$ for a quarter of a wavelength $\left(9.08^{\prime \prime}\right)$ to form a $\mathrm{ZO}=33 \Omega$ matching section between the garnet filled region and the standard $\mathrm{Z} 0=50 \Omega$ port of the quad hybrid. The OFHC copper center conductor/garnet cylinder is assembled using a shrink fit technique where the center conductor is first cooled to liquid nitrogen temperature and then inserted into the garnet cylinder. The phase shifter coaxial line outer conductor is a standard $1.625^{\prime \prime} \mathrm{OD}, 50 \Omega$ coaxial line (1.527" ID) whose OD has been reduced to $1.567^{\prime \prime}$ over the $9^{\prime \prime}$ length closest to the short. Both this $9^{\prime \prime}$ section and the bottom copper shorting plate have a $0.0197^{\prime \prime}$ wide slot machined through the copper to reduce eddy current effects. The shorted end of the phase shifter containing the garnet cylinder is surrounded by a solenoid wound from 47 turns of 12 AWG stranded copper THHN wire on a 1.812" OD G10 form. A magnetic flux return made from $0.75^{\prime \prime}$ thick ferrite blocks (TCI Ceramics material G4) helps to increase the phase shift range.

The larger version phase shifter uses a 3.0" OD x $0.65^{\prime \prime}$ ID x 5" long AL-400 garnet cylinder with a $Z_{0}=46 \Omega$ matching section. Here also, the outer conductor has a $0.020^{\prime \prime}$ thick wall and a $9^{\prime \prime}$ long slot. The biasing solenoid is 62 turns of 12 AWG wire on a 3.316" OD G10 form with a G4 ferrite flux return. The larger phase shifters are filled with $\mathrm{SF}_{6}$ to prevent sparking. To contain the $\mathrm{SF}_{6}$ which leaks through the slot, A G10 form fits around the phase shifter and is bolted to a flange on the shifter using an o-ring seal. A photograph of the various phase shifter components is shown in Fig. 田.

Each solenoid is independently powered by a 300V, 300A, two quadrant switching supply with a switching frequency of $250 \mathrm{kHz}$. A Praeg [9] style filter on the supply output, set to roll off at $40 \mathrm{kHz}$, is necessary to reduce the $250 \mathrm{kHz}$ ripple that would otherwise be seen on the vector modulator outputs. 


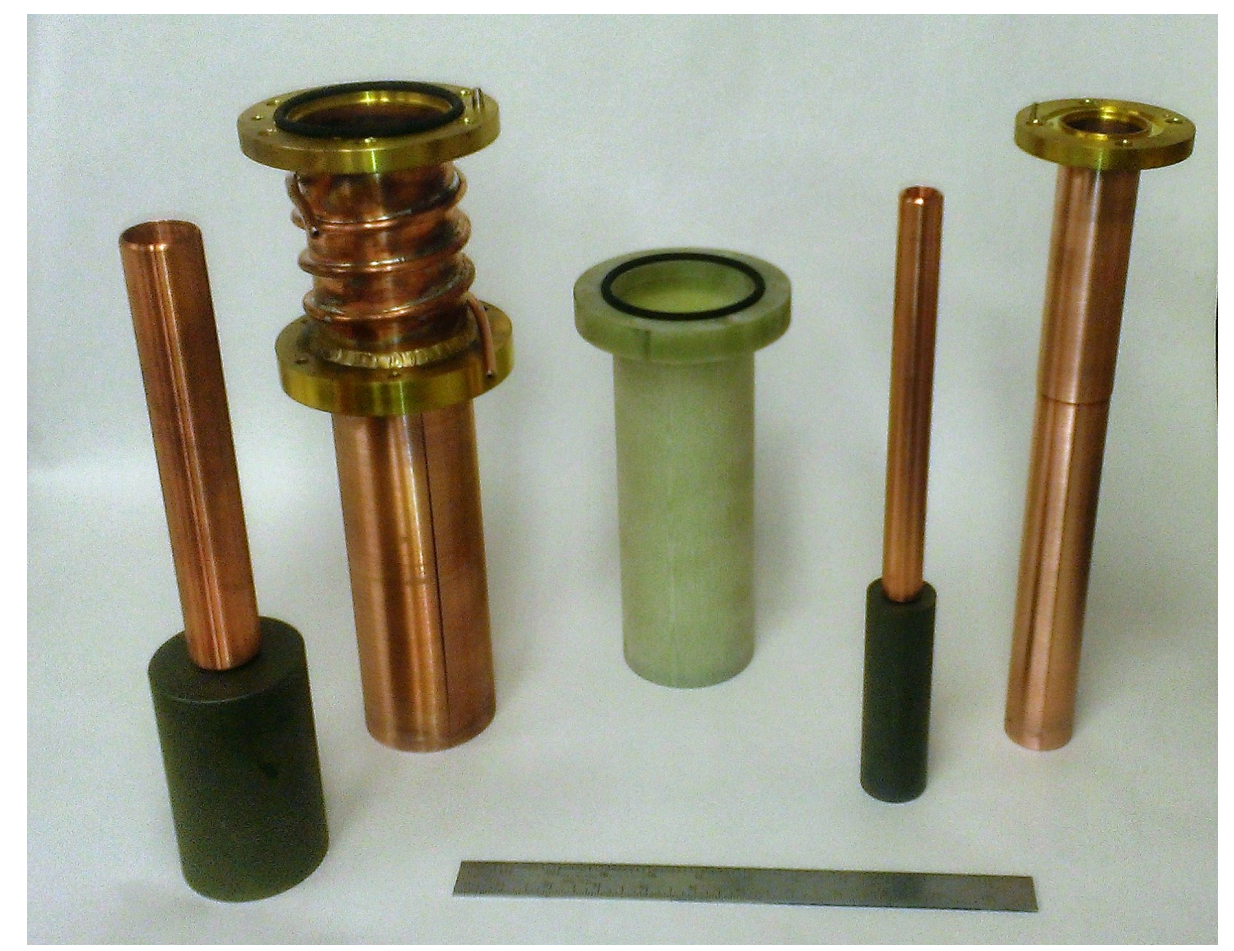

Figure 4. A photograph of the various phase shifter parts. From left to right, the $500 \mathrm{~kW}$ center conductor, outer conductor, $\mathrm{G} 10$ container for $\mathrm{SF}_{6}, 75 \mathrm{~kW}$ center conductor and outer conductor.

\subsection{Low Power Measurements}

\subsubsection{Phase Shift and Losses}

Fig. 5 shows low power (network analyzer) measurements of the round trip phase shifts and RF losses at $325 \mathrm{MHz}$ for both types of phase shifters, plotted as a function of the solenoid biasing field $H$. The maximum solenoid current for both cases was $300 \mathrm{~A}$ during the $4 \mathrm{~ms}$ pulse. The abrupt step in phase shift is due to ferromagnetic resonance in the garnet material. The resonance frequency as a function of magnetic field is given by [10]

$$
f_{0}=\gamma H_{s o l}+\left(N_{x}-N_{z}\right) M_{z}
$$

where $\gamma$ is the gyromagnetic ratio $(\approx 2.8 \mathrm{MHz} / \mathrm{Oe}), H_{s o l}$ is the field due to the solenoid (in the $z$ direction), $M_{z}$ is the magnetization in the $z$ direction, and $N_{x}$ and $N_{z}$ are the demagnetization factors in the $x$ and $y$ directions [11] [12] [13]. The demagnetization factors depend on the geometry of the sample and

$$
N_{x}+N_{y}+N_{z}=4 \pi
$$

For this case, due to symmetry, $N_{y}=N_{x}$. Since the smaller and larger garnets have different demagnetization factors, the $325 \mathrm{MHz}$ resonances occur at different applied fields.

Below and at resonance the RF losses are large which makes this region unsuitable for high power operation. Above 350-400 Oersted RF losses drop below $0.2 \mathrm{~dB}$ (typically $<0.1 \mathrm{~dB}$ ) and 


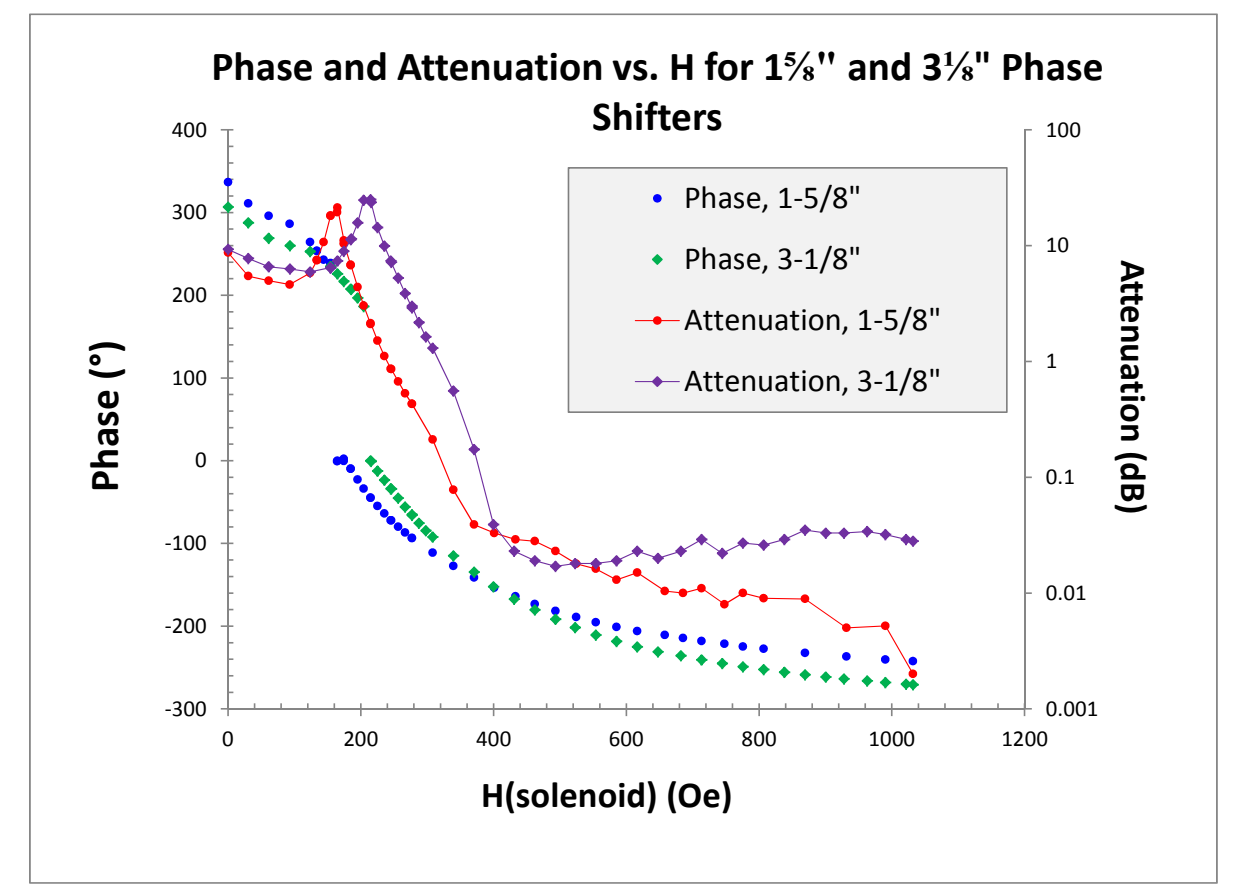

Figure 5. Round trip phase shift and attenuation, in a reflection (network analyzer $S_{11}$ ) measurement, for both types of phase shifters

high power operation becomes practical. Using $0.2 \mathrm{~dB}$ loss as an acceptable figure, the useable phase shift ranges are $131^{\circ}$ and $136^{\circ}$ for the small and large phase shifters, respectively.

\subsubsection{Effective Permeability}

Though the measurements in the previous sections are sufficient to characterize the phase shifters and the performance of the vector modulators, it was desirable to measure $\mu_{e}$, the effective permeability. Phase shift is proportional to $\sqrt{\varepsilon \mu_{e}}$, where $\varepsilon$ is the permittivity, so this yields a better understanding of the system and also lends itself well to future designs using the same type of material.

The equation of motion of the magnetization vector in the absence of losses is:

$$
\frac{d \mathbf{M}}{d t}=-\mu_{0} \gamma \mathbf{M} \times \mathbf{H}
$$

where $\mathbf{M}$ and $\mathbf{H}$, the magnetic field intensity, are sums of both DC and RF fields.

If the DC field is along the $z$-axis and the RF magnetic field is perpendicular to the $z$ axis, the equation of motion describes precession. Assuming that the garnet is biased to saturation so that $M_{z}$ is constant $\left(M_{z}=M_{s}\right)$, the solutions can be written in written in terms of the Polder tensor $[\mu]$, where $\mathbf{B}=[\mu] \mathbf{H}$ and

$$
[\mu]=\left[\begin{array}{ccc}
\mu & -j k & 0 \\
-j k & \mu & 0 \\
0 & 0 & \mu_{0}
\end{array}\right]
$$


where the elements are

$$
\mu=\mu_{0}\left(1+\frac{\omega_{0} \omega_{m}}{\omega_{0}^{2}-\omega^{2}}\right) \quad k=\mu_{0} \frac{\omega \omega_{m}}{\omega_{0}^{2}-\omega^{2}}
$$

and $\omega_{0}=\mu_{0} \gamma H_{\text {int }}, \omega_{m}=\mu_{0} \gamma M_{s}, \omega$ is the RF frequency and $H_{\text {int }}$ is the DC field in the garnet [14] [15] [16].

In the gaussian system this is written:

$$
\mu=1+\frac{4 \pi M_{s} H_{i n t} \gamma^{2}}{\gamma^{2} H_{i n t}^{2}-f^{2}} \quad k=\frac{4 \pi M_{s} \gamma f}{\gamma^{2} H_{i n t}^{2}-f^{2}}
$$

where $f$ is the frequency in $\mathrm{Hz}$ and $\gamma$ is $2.8 \mathrm{MHz} / \mathrm{Oe}$.

Note that the effective permeability $\mu_{e}$ is a function $\mathrm{H}_{\text {int }}$, the effective internal field inside of the garnet, which is not the same as $H_{e x t}$, the field generated by the solenoid.

$$
H_{\text {int }}=H_{\text {ext }}+\left(N_{x}-N_{z}\right) M_{z}
$$

In saturation, which is the region in which the phase shifters operate and the region in which Eq 3.8 is valid:

$$
H_{\text {int }}=H_{\text {ext }}+\left(N_{x}-N_{z}\right) M_{s}
$$

Determining the propagation constant, and thus the effective permeability $\mu_{e}$ which is of interest, requires solving Maxwell's Equations with $\mathbf{B}=[\mu] \mathbf{H}$, where $[\mu]$ is shown above. For a garnet loaded coaxial line, most authors resort to the approximation by Suhl and Walker [17], which is also summarized in von Aulock [18]. The result is

$$
\mu_{e}=\left(\mu^{2}-k^{2}\right) / \mu
$$

Where

- this is truly a parallel plane solution which is approximate as a solution for a coaxial line

- it is assumed that the garnet is saturated

- it is assumed that the garnet is lossless

A further approximation which is often made in Eq. 3.8 results in the ubiquitous $\mu_{e}=1+\frac{4 \pi M_{s}}{H_{\text {int }}}$, but this requires that $f \ll \gamma H_{\text {int }}$.

To measure $\mu_{e}$, one of the smaller, $1.5^{\prime \prime}$ garnet cores was placed in a coaxial line which was slightly longer than the garnet itself, which has a length $l$ of $5^{\prime \prime}$. The center conductor inside of the garnet was shorted to the outer conductor on one end. This formed a quarter wave resonator and the resonant frequency was measured as a function of solenoid bias. This was done by performing reflection $\left(S_{11}\right)$ measurements with a network analyzer capacitively coupled to the quarter wave fixture.

Once the frequency $f$ was measured for each bias, the product $\mu_{e} \varepsilon$ is known: 


$$
\sqrt{\mu_{e} \varepsilon}=\left(\frac{\lambda / 4}{l}\right)
$$

where $\lambda=c / f$. The solenoid used for the measurements described in this section is different from the solenoids used for the vector modulators. Its inner diameter is larger so it can accommodate the flange on the $\approx 1.625^{\prime \prime}$ OD quarter wave transmission line.

Since the OD of the garnet was approximately $0.042^{\prime \prime}$ smaller than the ID of the outer conductor, the manufacturer quoted value of the permittivity $(\varepsilon=14)$ could not be used. The short at one end of the resonator was removed, and using an HP 4263A RLC meter, the capacitance was measured at $100 \mathrm{kHz}$. Then, the effective permeability $\varepsilon$ is determined:

$$
C=\left(\frac{2 \pi \varepsilon \varepsilon_{0} l}{\ln (b / a)}\right)
$$

where $\varepsilon_{0}$ is the permeability of free space, $\mathrm{b}$ is the inner diameter of the outer conductor, and a is the outer diameter of the inner conductor.

This yielded a value of 10.65 . The capacitance was also measured at $2 \mathrm{MHz}$ using a vector impedance meter. In this case the corresponding values of $\varepsilon$ was 10.58 . Finally, a calculation based on the fixture dimensions and assuming $\varepsilon$ (garnet) $=14$ predicted that $\varepsilon=9.89$. The uncertainty on $\varepsilon$ is the determining factor in the uncertainty on the measurement of $\mu_{e}$. The error on $\varepsilon$ is taken to be the difference between the measurement with the RLC meter and the calculated value.

Since the goal is to compare measured values of $\mu_{e}$ with the theoretical prediction, it is necessary to also determine $N_{z}$, since this determines $H_{\text {int }}$. Two pieces of garnet without coaxial transmission line were stacked one on top of the other as shown in Fig 6, inside of the solenoid. The bottom piece of garnet had a slot filed in it, into which a magnetic field probe was placed. Magnetic field $B$ was measured as a function of solenoid magnetic field intensity $H_{\text {sol }}$. The results are shown in Fig 7 .

Since

$$
B_{z}=H_{s o l}-N_{z} M_{z}+4 \pi M_{z}
$$

A line fit to the linear portion of the plot where $M_{z}=M_{s}$ has a $y$-intercept of $\left(1-\frac{N_{z}}{4 \pi}\right) 4 \pi M_{s}$. Using the manufacturers quoted value of $4 \pi M_{s}=400$ gauss $( \pm 5 \%)$ gives $N_{z} / 4 \pi=0.42$.

An alternative measurement of $N_{z}$ was performed. Using a network analyzer reflection $\left(S_{11}\right)$ measurement, the gyromagnetic resonance frequency and $H_{s o l}$ were determined for the case where the resonance is near $325 \mathrm{MHz}$. The exact values were $f=324.875$ at $H_{\text {sol }}=154.8 \mathrm{Oe}$. Using Eq 3.1 with $M_{z}=M_{s}$ and $4 \pi M_{s}=400$ gauss, $N_{z} / 4 \pi=0.398$. Since the second measurement is more accurate, the latter value for $N_{z}$ is used in the calculation of $H_{\text {int }}$.

The measurements of $\mu_{e}$ vs. $H_{\text {int }}$ and the theoretical curve of Eq 3.8 are shown in Fig 8 . The error on $\mu_{e}$ is due entirely to how well $\varepsilon$ is known. Other sources of error are neglible. Note that when the material is not saturated, $M_{z} \neq M_{s}$ and Eq 3.8 is no longer valid. From Fig $\square$ it can be estimated that saturation occurs at approximately $H_{\text {sol }}=150$ Oe where $H_{\text {int }}$ is approximately 111 Oe. Below this region, the theoretical curve is represented by a dashed line. In addition, when not saturated, the determination of $H_{\text {int }}$ by Eq 3.7 is no longer valid since $M_{z} \neq M_{s}$. Since $M_{z}$ is not 


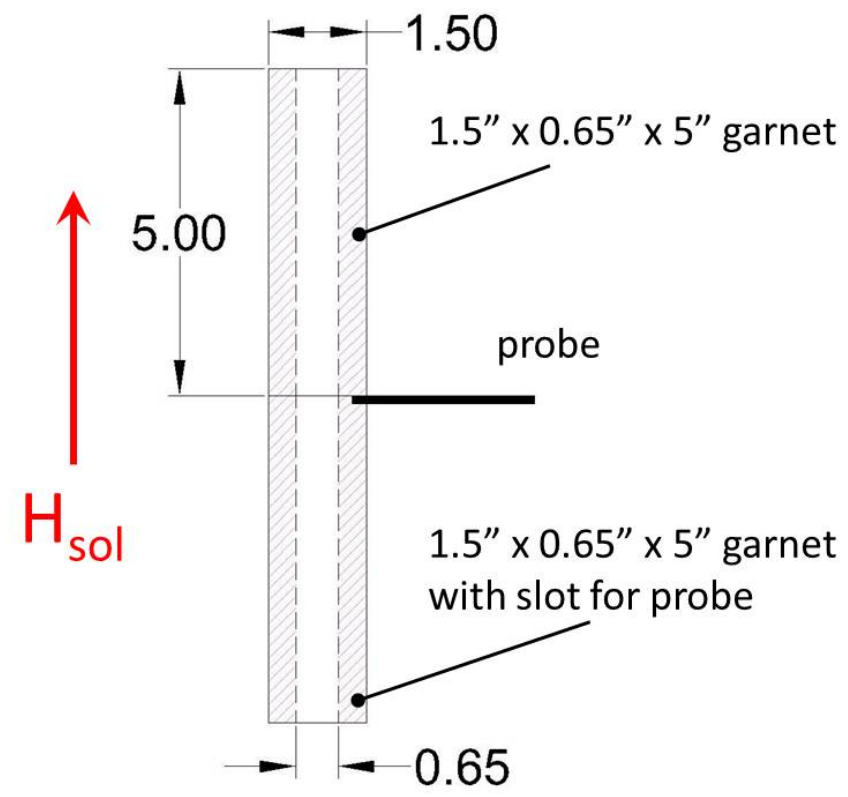

Figure 6. Sketch of the setup of the DC measurement of $B$ (garnet) vs. $H_{\text {sol }}$. Two pieces of garnet are placed end to end inside of the solenoid. The lower piece of garnet has a slot filed in it, into which the magnetic field probe is inserted.

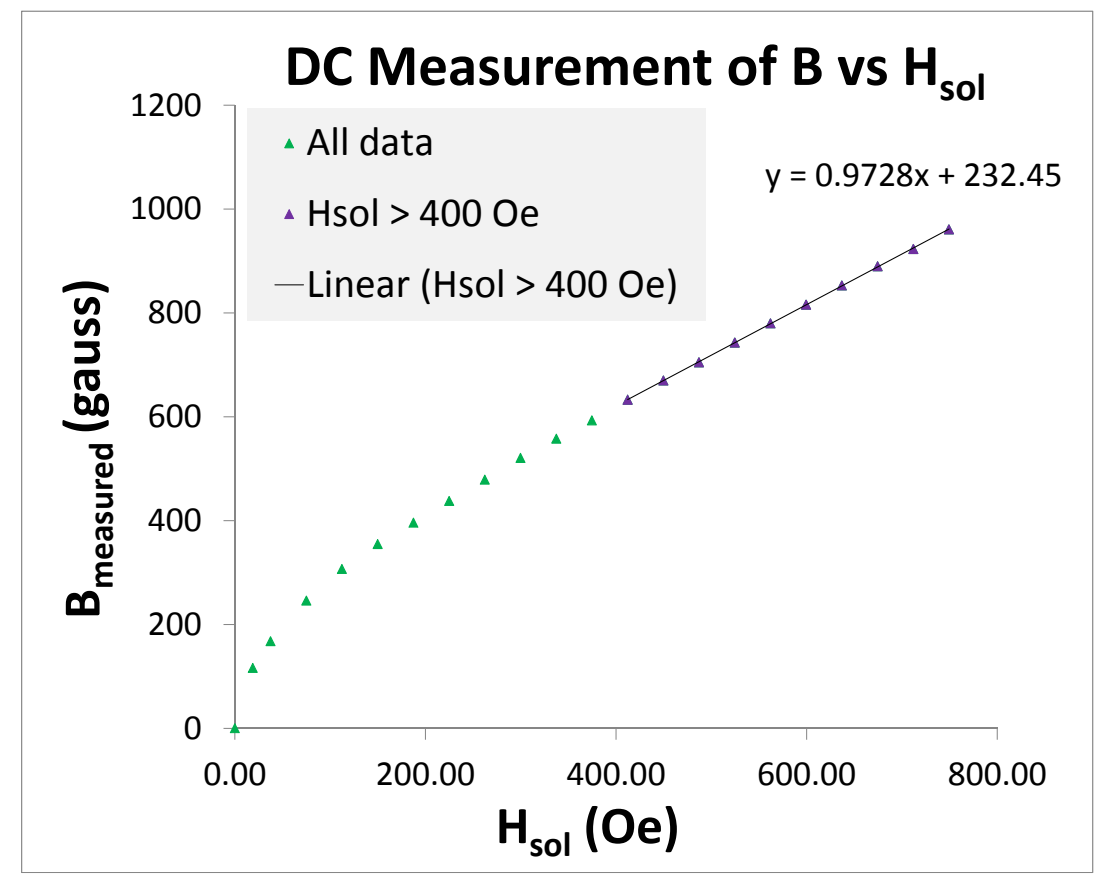

Figure 7. DC measurement of $B$ (garnet) vs. $H_{\text {sol }}$. The linear part of the data is fit to a line. The $y$-intercept of the line is $\left(1-\frac{N_{z}}{4 \pi}\right) 4 \pi M_{s}$

known here, large error bars on $H_{\text {int }}$ are used to illustrate the fact that it can be between 0 and $M_{s}$. The other $H_{\text {int }}$ error bars reflect that the measurement precision for $H_{\text {sol }}$ and thus $H_{\text {int }}$ is $5 \%$. 
One caveat which was mentioned earlier is that the theoretical curve for $\mu_{e}$ as a function of $H_{\text {int }}$ is not valid in the case where the garnet is lossy. An expression for $\mu_{e}$ in the presence of losses is presented in [18]. In this case there is one more free parameter determining the shape of the curve. This is $\Delta H$, the gyromagnetic resonance linewidth. The manufacturer quoted value is $45 \mathrm{Oe}$. In this case the theoretical curves with and without losses are indistinguishable in the regions in which they are valid.

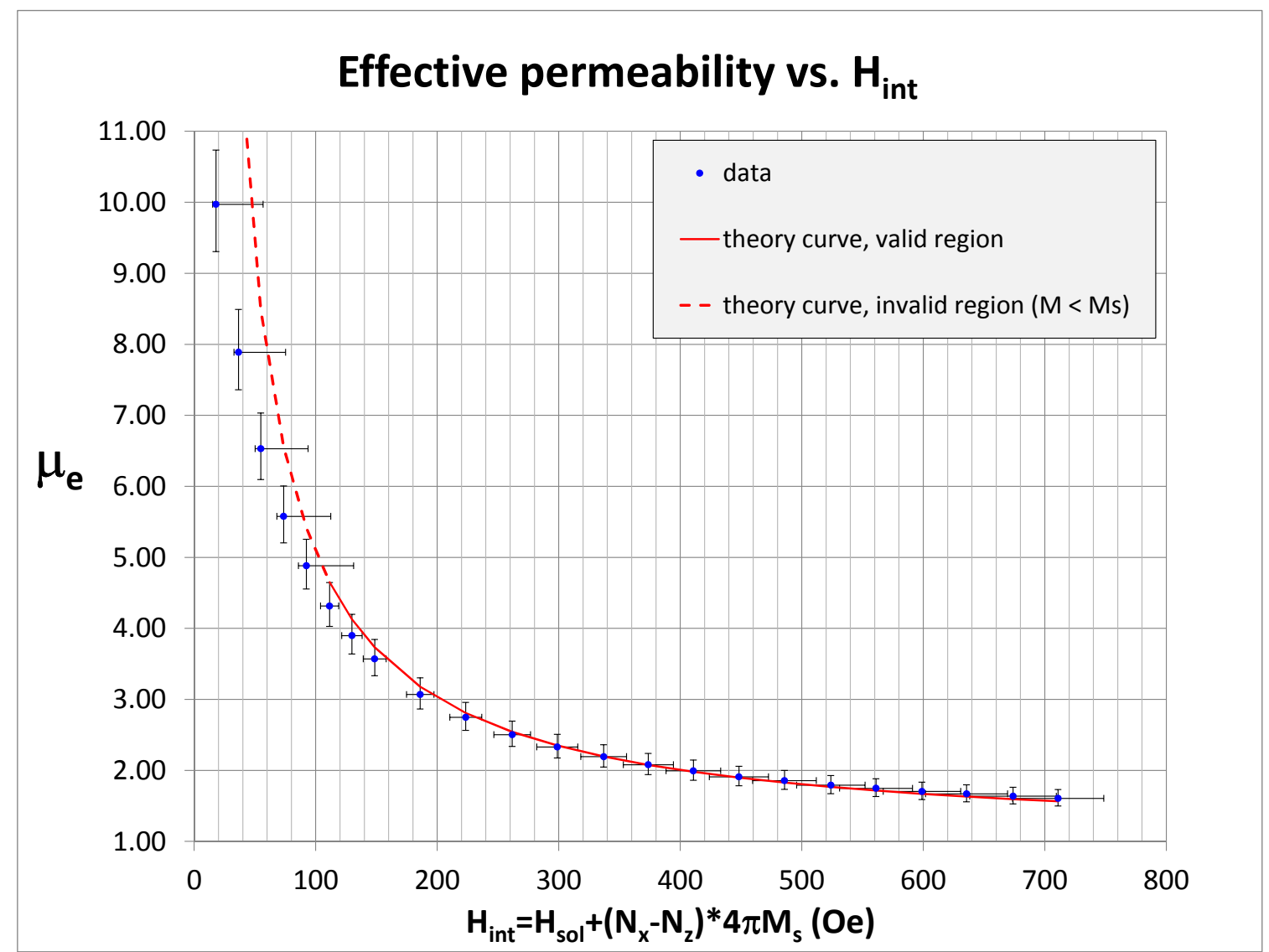

Figure 8. Measurements and theoretical predictions of effective permeability $\mu_{e}$ as a function of $H_{\text {int }}$. The theoretical prediction is not valid for $M_{z}<M_{s}$, so it is shown as a dotted line. Large error bars on the values of $H_{\text {int }}$ below saturation reflect the fact that $H_{\text {int }}$ is not known well, since $M_{z}$ is not known. 


\subsubsection{Response Time}

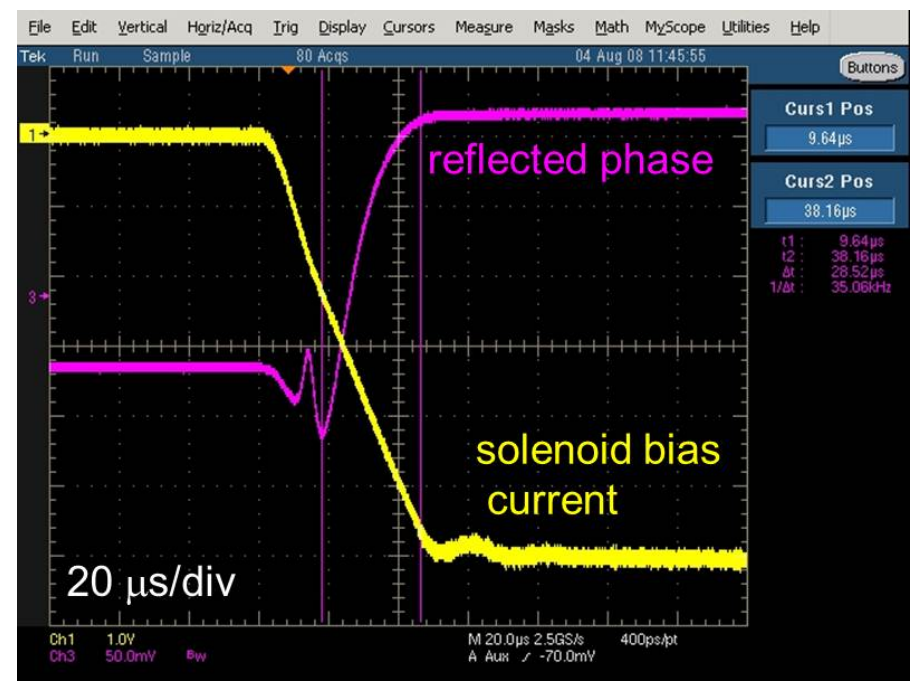

Figure 9. Phase shifter response at $325 \mathrm{MHz}$ to a step in solenoid current, as a function of time. Reflected (output) phase is shown on Channel 3 , at $30^{\circ}$ per division. The solenoid current is shown on Channel 1, at 50 A per division. The time scale is $20 \mu$ s per division.

Fig. 9 shows the time response of the smaller type phase shifter. In this case, the current program is a step function with a risetime of less than $1 \mu \mathrm{s}$. The corresponding solenoid current response (from 0 to $300 \mathrm{~A}$ ) and phase are shown on Channels 1 and 3 of a scope. The rate of rise of the solenoid current is limited by the $300 \mathrm{~V}$ maximum output of the supply and the $\approx 45 \mu \mathrm{H}$ inductance of the solenoid. The measured phase shift was obtained with an HP 86205A directional bridge and HP 10514A mixer, used as a phase detector at $325 \mathrm{MHz}$, comparing the input and reflected RF signals. Above resonance the average phase slew rate is $\approx 6^{\circ} / \mu \mathrm{s}$

The small signal $\left( \pm 10^{\circ}\right)$ frequency response for the two types of phase shifters, centered at midrange, was also measured. The sinusoidal current in the solenoid (with the DC level at midrange) was varied from 0.5 to $75 \mathrm{kHz}$. A $325 \mathrm{MHz} \mathrm{RF}$ signal is input to the phase shifter, and the phase of the reflected signal is measured using a directional coupler and mixer. This phase changes as the current in the solenoid is modulated, and the higher the frequency of the current in the solenoid, the more the phase shift lags. The lag time, normalized to frequency on the $\mathrm{x}$-axis (the "phase of the phase") is plotted as a function of frequency in Fig 10. For example, a $0.5 \mathrm{kHz}$ sinusoidal modulation of the solenoid bias current results in the phase of the modulated $325 \mathrm{MHz}$ signal lagging the current waveform in the solenoid by $56 \mathrm{~ms}$, or $56 \mathrm{~ms} \times 360^{\circ} / 0.5 \mathrm{kHz}=10.08^{\circ}$. These measurements were performed both open loop and with feedback. The feedback error signal was obtained by comparing the measured reflected phase to a reference. The error signal was then amplified and summed back into the bias solenoid current program. The small shifter open loop bandwidth is $\approx 15 \mathrm{kHz}$ which was extended to $>35 \mathrm{kHz}$ with the phase feedback. 


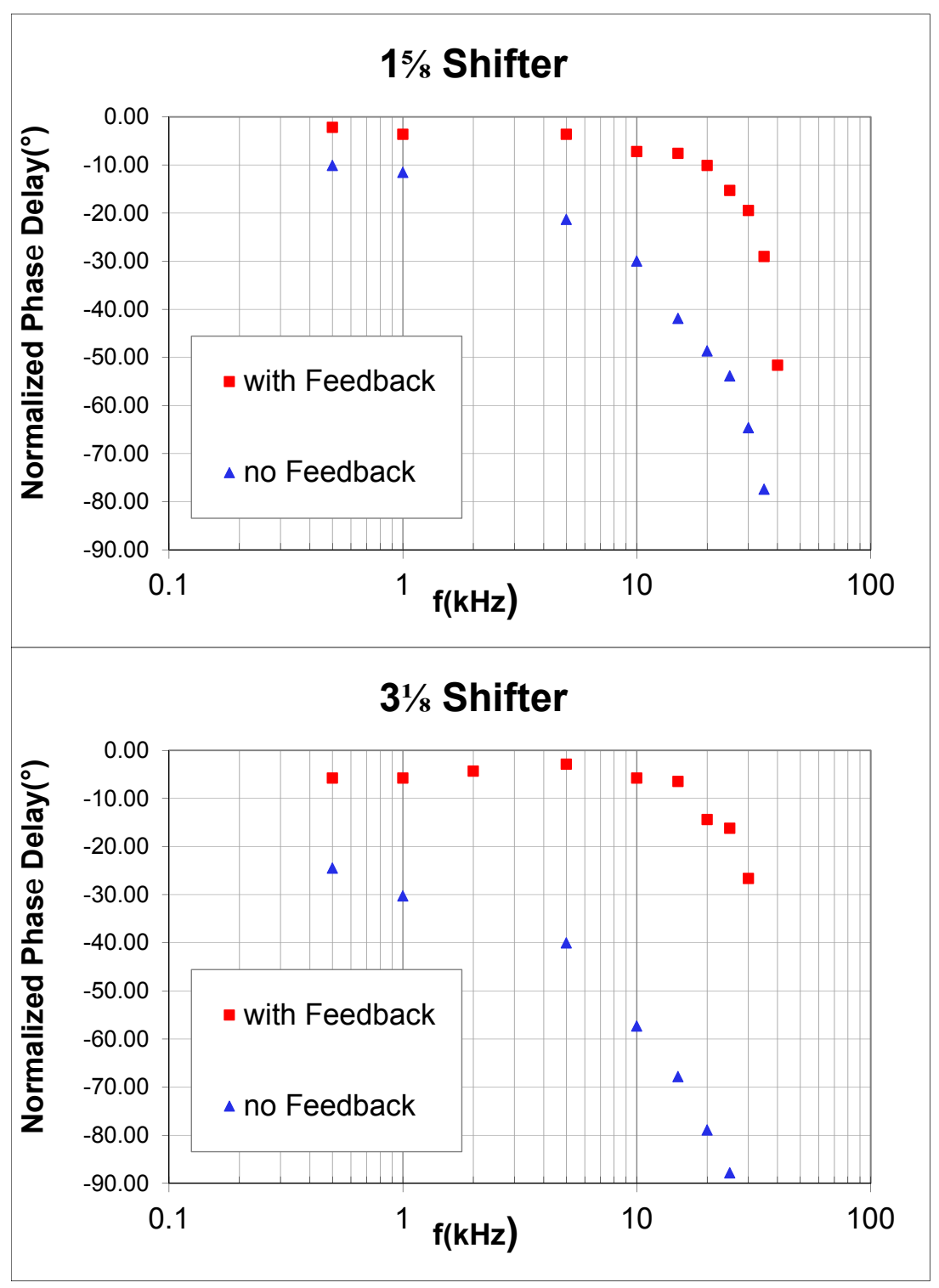

Figure 10. Phase shifter small signal response with and without feedback.

\section{Vector Modulator High Power Operation}

\subsection{Preparation}

Approximately 30 of the smaller type of phase shifters have been made. Due to variation in production lots of the AL-400 and other unknown factors, there is a variation in reflected phase from one phase shifter to another, even when the solenoid field is the same. In order to achieve similar response curves for all of the vector modulators, and given the variation in outputs, the response for each phase shifter was measured. Pairs which were most alike were then selected to be used in the same vector modulator. To perform this measurement quickly, a solenoid which was twice the length of the usual solenoid for operation was constructed. Each phase shifter was inserted into 
this solenoid opposite a reference phase shifter. A network analyzer measuring reflected phase was triggered to sweep (in time) along with the current ramp from 0 to maximum. The reflected phase was measured at nine different times during the ramp, for the reference phase shifter along with each phase shifter, and the difference was calculated. The results are shown in Fig [1]. The phase shifters with the most similar phase response were paired. The variation is large near resonance (the third point), however, the phase shifters are not operated in this region due to high RF losses

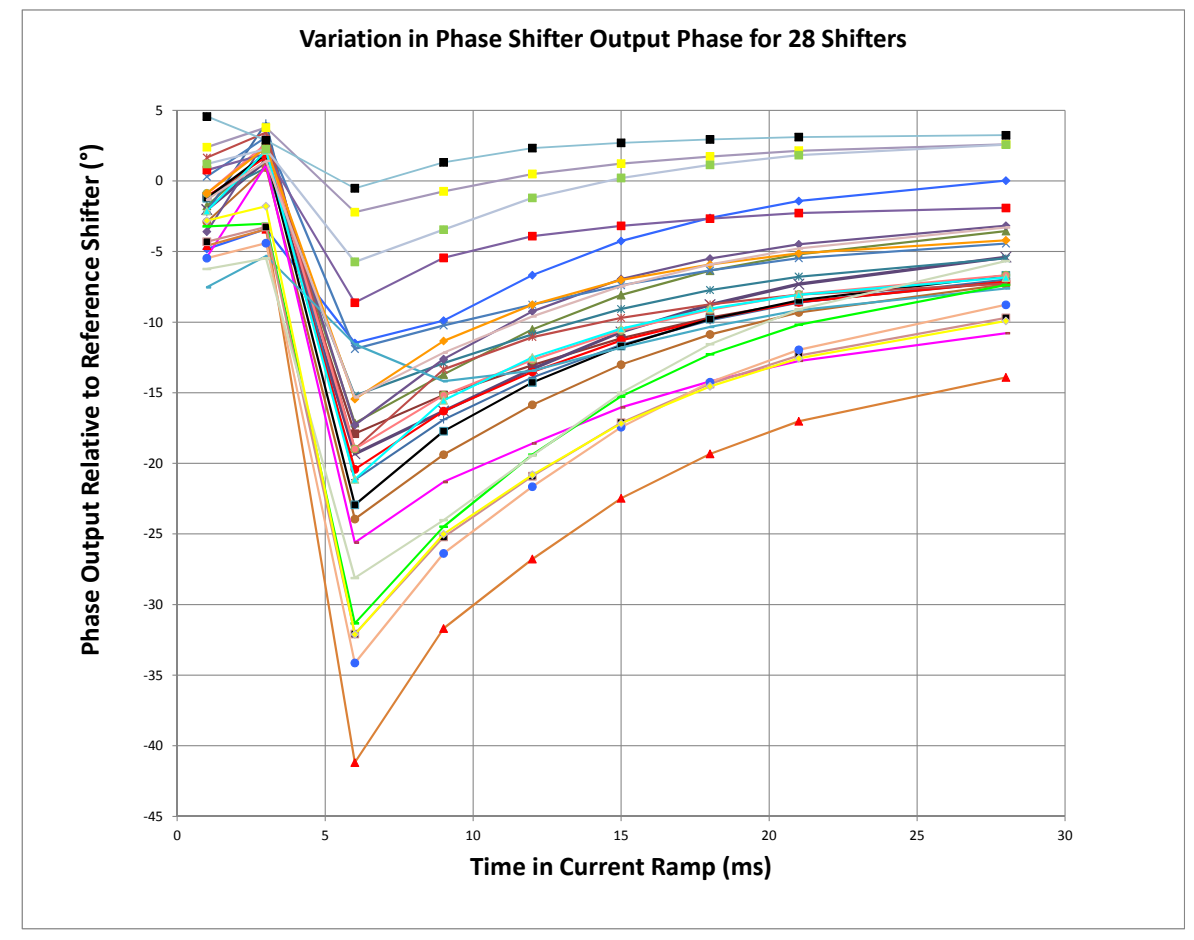

Figure 11. Variation in phase shifter phase shift, relative to one reference phase shifter. Each curve shows the data for one of 28 shifters. The phase is recorded and the current is swept from 0 to the maximum in a period of $28 \mathrm{~ms}$. Network analyzer phase measurements are made at nine times during this sweep, corresponding to the points on the $\mathrm{x}$ axis.

\subsection{Standalone Operation}

Fig. 12 illustrates the amplitude modulation capability of the $75 \mathrm{~kW}$ vector modulator during a $2 \mathrm{~ms}$ RF pulse without any phase feedback. With a constant $50 \mathrm{~kW}$ input power to the vector modulator, the output power is stepped from $25 \mathrm{~kW}\left(\Delta \phi_{2}-\Delta \phi_{3}=90^{\circ}\right)$ to the full $50 \mathrm{~kW}\left(\Delta \phi_{2}-\Delta \phi_{3}=0^{\circ}\right)$. During the first portion of the pulse, the $25 \mathrm{~kW}$ of unwanted power is directed back towards the circulator at the output of the $2.5 \mathrm{MW}$ klystron where it is absorbed in the circulator load. Both the $75 \mathrm{~kW}$ and $500 \mathrm{~kW}$ phase shifters have successfully operated at their design power levels.

Fig. 13 shows the magnitude of the electric field in an RTCH cavity with a vector modulator attached to the cavity with $6 \mathrm{~kW}$ of forward power from the $2.5 \mathrm{MW}$ klystron. One phase shifter on the vector modulator has its solenoid biased to $80 \mathrm{~A}$ for the entire pulse; the other phase shifter is biased to its maximum ( $\mathrm{I}=320 \mathrm{~A}$ ) for the first half of the pulse and to $80 \mathrm{~A}$ for the second half of the pulse. The corresponding change in the amplitude of the field in the cavity is $13 \mathrm{~dB}$. 


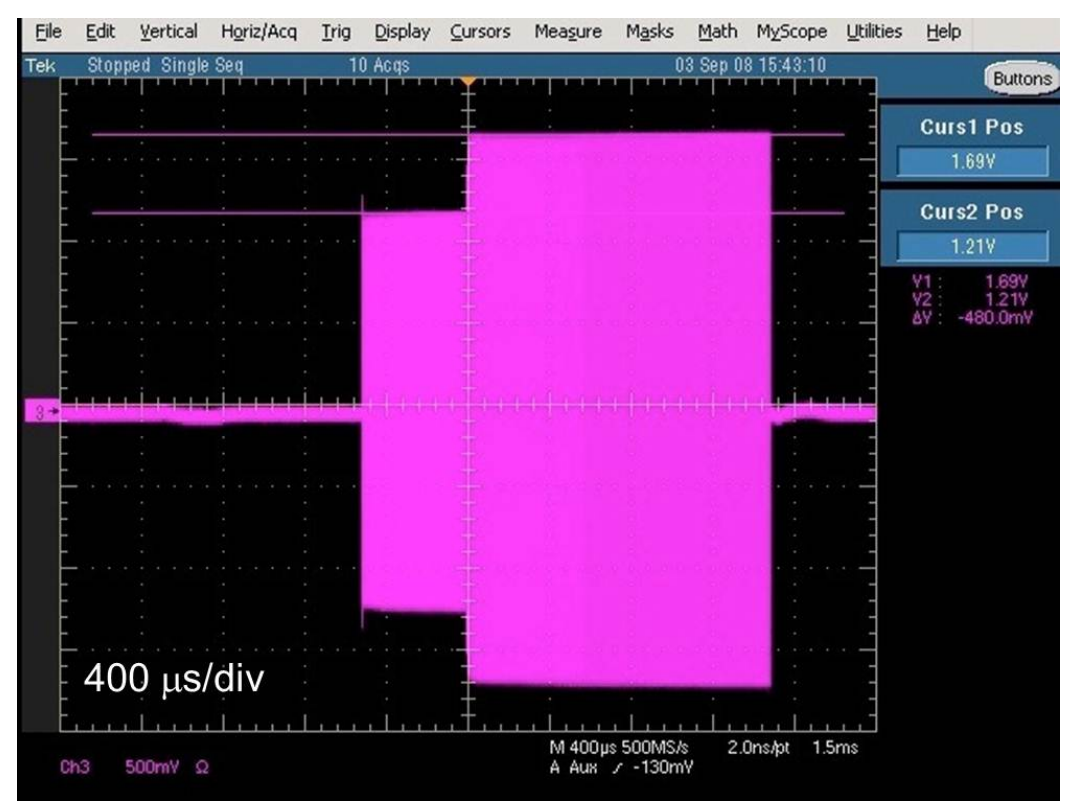

Figure 12. Vector modulator amplitude modulation at 25 to $50 \mathrm{~kW}$.

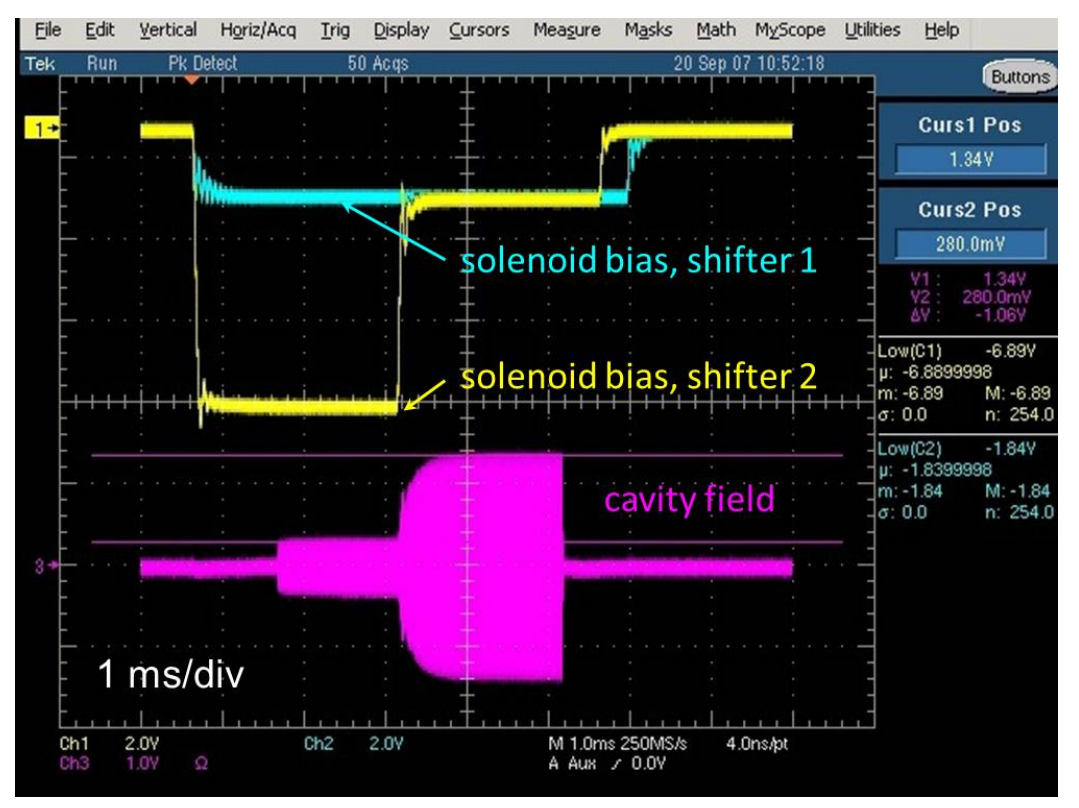

Figure 13. Vector modulator with an RTCH cavity showing cavity field response.

\subsection{Vector Modulators with Beam in The Six Cavity Test}

In the HINS linac for The Six Cavity Test, proton beam is generated by a $50 \mathrm{kV}$ duoplasmatron source followed by two solenoids for focusing. It is then accelerated to $2.5 \mathrm{MeV}$ by a $325 \mathrm{MHz}$ RFQ, longitudinally focused by a buncher cavity, accelerated to $3.4 \mathrm{MeV}$ by four RTCH triple spoke cavities and then focused again by one final buncher cavity. The beam is focused transversely by quadrupole triplets. Instrumentation and diagnostics are placed along and at the end of the beamline. The beam current was $\approx 7 \mathrm{~mA}$. 


\begin{tabular}{|l|c|c|c|c|}
\hline Description & \multicolumn{2}{|c|}{ VM Control OFF } & \multicolumn{2}{c|}{ VM Control ON } \\
& $\begin{array}{c}\text { Mag } \\
(\%)\end{array}$ & $\begin{array}{c}\text { Phase } \\
\left({ }^{\circ}\right)\end{array}$ & $\begin{array}{c}\text { Mag } \\
(\%)\end{array}$ & $\begin{array}{c}\text { Phase } \\
\left({ }^{\circ}\right)\end{array}$ \\
\hline RFQ & 0.021 & 0.015 & 0.021 & 0.015 \\
Buncher 1 & 0.605 & 0.945 & 0.142 & 0.089 \\
Cavity 1* & 2.254 & 0.435 & 1.664 & 0.647 \\
Cavity 2 & 1.737 & 1.200 & 0.203 & 0.209 \\
Cavity 3 & 1.070 & 1.434 & 0.201 & 0.145 \\
Cavity 4 & 0.543 & 1.887 & 0.159 & 0.149 \\
Buncher 2 & 0.457 & 2.314 & 0.190 & 0.113 \\
\hline
\end{tabular}

*VM control dynamic range limit reached.

Table 1. RMS cavity field magnitude and phase variation across the pulse with RFQ feedback, with and without the vector modulator control loop active. The results are slightly worse for Cavity 1 due to lack of available RF input power overhead given the power splitting scheme.

For this test, the RFQ vector modulator was not used. Instead, the klystron output RF is split equally between the RFQ and the rest of the cavities, each of which has a vector modulator on its input. The half of the power to the remaining six cavities is then further split (unequally) to supply the required power to each cavity. The low level RF (LLRF) control system regulates the phase and amplitude of the RF field vectors of the RFQ and the six cavities [19]. This makes use of a traditional wide-band proportional and integral feedback control loop with the klystron and the RFQ. This removes most of the error due to voltage variations of the klystron modulator and beam current variation. For the six cavities, there are several factors to which the vector modulators must respond. These are constant errors in amplitude and phase, cavity frequency drift due to temperature, and variations in beam loading. Field errors in the cavities are corrected by an adaptive feed-forward system which controls the vector modulators. This system has two parallel loops, one operating on the average (DC) error, and the other working on the AC component of the error. Studies have shown that amplitude is regulated to better than $1 \%$, and phase to better than 1 degree, both within the pulse and from pulse to pulse. The details of the performance are shown in Table 1[四 $]^{1}$.

\section{Summary}

A new type of vector modulator using perpendicularly biased garnet has been developed. Measurements of various quantities associated with the component phase shifters and the vector modulators as a whole have been presented. Successful high power operation of the vector modulators, first, as a standalone system, and later, in a test linac with beam, has been demonstrated. Though the vector modulators discussed here were developed for a specific purpose, the concept can be easily extended to include a wide range of applications.

\footnotetext{
${ }^{1}$ Many thanks to J. Steimel et al.
} 


\section{Acknowledgments}

We would like to thank the Fermilab Accelerator Division EE Support Group, specifically, B. Claypool. S. Hays, and H. Pfeffer for providing the solenoid pulsed power supplies used in this work. In addition, we would like to thank everyone involved in HINS, including commissioning, operation and the Six Cavity Test. We would also like to thank the Accelerator Division LLRF group for their work on the LLRF control system for the operation of the vector modulators in the Six Cavity Test.

This work is supported by Fermi Research Alliance, LLC under Contract No. DE-AC0207CH11359 with the United States Department of Energy.

\section{References}

[1] R. Webber, G. Apollinari, Overview and status update of the Fermilab HINS Linac R\&D program, in: Proc. PAC 2009, Vancouver, BC, Canada, 2009, p. 4902.

[2] P. Ostroumov, Physics design of the 8 GeV H- Linac, New J. Phys. 8 (2006) 281.

[3] L. Ristori, et al., Fabrication and test of the first normal-conducting crossbar H-type accelerating cavity at Fermilab for Hins, in: Proc. PAC 2007, Albuquerque, NM, USA, 2007, pp. 2292-2294.

[4] J. Steimel, et al., The six-cavity test - demonstrated acceleration of beam with multiple RF cavities and a single klystron, in: Proc. IPAC 2012, New Orleans, LA, USA, 2012, pp. 3877-3879.

[5] A. S. Boxer, et al., A high-power coaxial ferrite phase shifter, IEEE Trans Microwave Theory Tech 9 (6) (1961) 577.

[6] A. S. Boxer, R. S. McCarter, Coaxial ferrite phase shifter for high power applications, J. Appl. Phys. 33 (03) (1962) 1263-1264.

[7] R. Madrak, D. Wildman, New materials and designs for high power, fast phase shifters, in: Proc. LINAC2006, Knoxville, TN, 2006, pp. 829-831.

[8] J. Wilson, et al., Development of high-power RF vector modulator employing TEM ferrite phase shifters, in: Proc. LINAC2006, Knoxville, TN, 2006, pp. 451-43.

[9] W. F. Praeg, A high-current low-pass filter for magnet power supplies, IEEE Trans. Industrial Electronics and Control Inst. IECI-17 (1970) 16.

[10] C. Kittel, On the theory of ferromagnetic resonance absorbtion, Phys. Rev. 73 (62) (1948) 155-161.

[11] H. Knoepfel, Magnetic Fields, John Wiley and Sons, Inc., New York, 2000, pp. 10-11,13-15.

[12] S. Chikazumi, Physics of Ferromagnetism, Oxford University Press, Oxford, 2002, pp. 13-16.

[13] R. M. Bozorth, D. M. Chapin, Demagnetization factors of rods, Journal of Applied Physics 321 (1942) 1306-1315.

[14] Ü. Özgur, et al., Microwave ferrites, part 1: Fundamental properties, Journal of Materials Science: Materials in Electronics 20 (9) (2009) 789-834.

[15] D. Pozar, Microwave Engineering, Third Edition, John Wiley and Sons, Inc., 2005.

[16] H. Suhl, L. R. Walker, Topics in guided wave propagation through gyromagnetic media, part I, Bell Sys. Tech J 33 (1954) 1133-1194. 
[17] H. Suhl, L. R. Walker, Topics in guided wave propagation through gyromagnetic media, part III, Bell Sys. Tech J 33 (1954) 579-659.

[18] W. H. von Aulock, C. E. Fay, Linear Ferrite Deviced for Microwave Applications, Academic Press, Inc., New York, 1968, p. 30.

[19] P. Varghese, et al., Performance of ferrite vector modulators in the LLRF system of the Fermilab HINS 6-cavity test, in: Proc. LINAC2012, Tel-Avis, Israel, 2012, pp. 810-812. 